\title{
Prognostic significance of CKS2 and CD47 expression in patients with gastric cancer who underwent radical gastrectomy
}

(i)A short informative title:

Prognostic significance of CKS2 and CD47 in gastric cancer patients

(ii)The full names of the authors:

Yang Zhou, Jing Zeng ${ }^{\star}$, Haoran Zhuang, Wei Zhou,Keyan Wu, Weigan Shen*

\&:Mrs Jing Zeng contributed to the work equllly and should be regarded as co-first authors

(iii)All author's institutional affiliation:

Department of Cell Biology, School of Medicine of Yangzhou University, Yangzhou, 225000, People's Republic of China

(iV )Corresponding author information;

Dr. Weigan Shen

Department of Cell Biology, School of Medicine of Yangzhou University, Yangzhou, 225000, People's Republic of China.

E-mail: zyjsyy666@aliyun.com

(v)Disclosure statement ;

We have no financial relationships to disclose. 
Objective: To investigate the protein expression levels of cyclin-dependent kinase subunit 2 (CKS2) and cluster of differentiation (CD) 47 in gastric cancer (GC) and their clinical significances. Methods: A total of $126 \mathrm{GC}$ patients who underwent radical resection were selected as study subjects. Additionally, 32 patients with benign gastric tumor, 42 patients with low-grade intraepithelial neoplasia (LGIEN), and 49 patients with high-grade intraepithelial neoplasia (HGIEN) who underwent surgery were selected as the control groups. Immunohistochemistry was used to detect the expression of CKS2 and CD47 in surgical specimens. We statistically analyzed the clinical significance of the expression of the two factors. Results: (1) The positivity rates for CKS2 in benign gastric tumor tissue, LGIEN tissue, HGIEN tissue, and GC tissue gradually increased, i.e., $6.3 \%$ (2/32), 30.9\% (13/42), 38.8\% (19/49), and 60.3\% (76/126), respectively, and the positivity rates for CD47 were $18.8 \%$ (6/32), 38.1\% (16/42), 46.9\% (23/49), and 65.9\% (83/126), respectively. (2) High expression of CKS2 and CD47 were associated with tumor diameter, Lauren classification, number of lymph node metastases, and TNM stage. In addition, the immunohistochemical scores for CKS2 and CD47 were positively correlated ( $\mathrm{r}=0.625, \mathrm{P}=0.000)$. (3) The median follow-up time of 126 patients was 46.5 months, and the overall survival rate was $40.5 \%$ (51/126). Survival analysis showed that compared with that in the CKS2 (-) group, the overall survival rate for patients in the CKS2 (+) group was significantly worse $(25.0 \%$ vs $64.0 \%$, $\left.\chi^{2}=15.67, \mathrm{P}=0.000\right)$ and that compared with the CD47 (-) group, the CD47 (+) group had significantly worse overall survival $\left(30.1 \%\right.$ vs $\left.60.5 \%, \chi^{2}=15.67, \mathrm{P}=0.000\right)$. (4) The overall survival rates of CKS2(+)CD47(+) group, CKS2(+)CD47(-) group, CKS2(-)CD47(+) group, and CKS2 (-)CD47 (-) group were 20.0\%(13/65), 58.3\%(7/12), 57.1\%(8/14), 65.7\% (23/35), respectively, the prognosis of patients in CKS2(+)CD47(+) group was significantly poor. Conclusion: High expression levels of CKS2 and CD47 were closely related to the occurrence of GC and can be used as independent risk factors to assess the prognosis of patients.

Key words: Gastric cancer; Cyclin-dependent protein kinase; Cluster of differentiation (CD) 47; Prognosis

Gastric cancer (GC) is one of the most common malignant tumors in the world. In 2012, there were a total of 951,000 new GC patients and 723,000 GC-related deaths worldwide, with morbidity 
and mortality rates ranking fifth and third, respectively, among all malignant tumors ${ }^{[1]}$. In China, GC is the second most common malignant tumor and seriously threatens the health and life of people ${ }^{[2]}$. Despite continuous improvements in the diagnosis and treatment of GC, the overall prognosis is still not satisfactory, with a 5-year survival rate of approximately $30-60 \%{ }^{[3]}$. Surgical resection combined with chemotherapy is one of the conventional treatments for GC, and the application of a series of chemotherapeutic drugs and molecular targeted drugs, such as oxaliplatin, 5-fluorouracil, and herceptin, improves the clinical outcomes of GC patients. However, the prognosis of patients with GC is still not optimistic due to the toxicity/side effects of the drugs ${ }^{[4]}$. Therefore, it is necessary to seek for new molecular therapeutic targets and investigate their correlations with the prognosis of patients with GC to further optimize precise treatment for GC patients. Cyclin-dependent kinase subunit 2 (CKS2) is a member of the cyclin-dependent kinase subunit family, it is localized on human chromosome 9q22.2, and its abnormal expression is closely related to the occurrence and development of a variety of cancers ${ }^{[5]}$. During the development of malignant tumors, CKS2 regulates the activity of the cyclin-dependent kinase (CDK)1-cyclin B complex and thus participates in multiple processes, such as cell cycle regulation, tumor invasion, metastasis, and apoptosis ${ }^{[6]}$. However, the relationship between CKS2 protein and the prognosis of GC patients remains to be established. Cluster of differentiation (CD) 47, also known as integrin-associated protein (IAP), is a transmembrane glycoprotein with a molecular weight of 50 $\mathrm{kD}^{[7]}$. CD47 is expressed not only in tumor cells but also in normal cells to some extent. Studies have shown high CD47 expression in leukemia cells and a variety of solid tumors ${ }^{[8]}$. However, in GC patients, whether CD47 expression is a risk factor affecting prognosis requires more experimental studies. This study assessed the protein expression levels of CKS2 and CD44 in GC tissues by immunohistochemistry, and clarified their potential as independent prognostic factors and molecular therapeutic targets for the treatment of GC.

\section{Materials and methods}

\subsection{Patients}

GC tissue samples were retrospectively collected from 126 patients (stages IB to III) who underwent R0 gastrectomy with extensive node dissection (D2) and adjuvant chemoradiation 
therapy from 2013 to 2018 at Yancheng First People's Hospital, China. The inclusion criteria were as follows: (1) patients who were initially diagnosed and treated; (2) patients who were diagnosed with primary GC and underwent radical surgical resection; (3) no radiochemotherapy before surgery; (4) histopathological type, adenocarcinoma; (5) age <80 years; and (6) complete clinical and pathological data. The exclusion criteria were as follows: (1) history of other malignancies; (2) GC patients with emergency operation due to sudden obstruction, severe bleeding, and perforation; and (3) perioperative death, for example, pulmonary embolism caused by deep vein thrombosis, septic shock caused by anastomotic fistula, and multiple organ failure. In total, $126 \mathrm{GC}$ patients were included in the study: 78 males, with an average age of $(64.8 \pm 12.7)$ years, and 48 females, with an average age of $(59.7 \pm 10.3)$ years. The clinicopathological data of the patients, including age, gender, tumor size, depth of invasion, degree of differentiation, histological type, Lauren classification, lymph node metastasis, and tumor-node-metastasis (TNM) stage (according to the 8th Edition of American Joint Committee on Cancer (AJCC) Cancer Staging Manual), were obtained through the electronic medical record system. Additionally, 32 patients with benign gastric tumor, 42 patients with low-grade intraepithelial neoplasia (LGIEN), and 49 patients with high-grade intraepithelial neoplasia (HGIEN) who underwent surgical treatment during the same period were selected as the control group. In the benign gastric tumor group, there were 22 males, with an average age of $(50.1 \pm 5.3)$ years, and 10 females, with an average age of $(43.6 \pm 9.8)$ years; in the LGIEN group, there were 26 males, with an average age of $(55.6 \pm 11.8)$ years, and 16 females, with an average age of $(54.6 \pm 8.8)$ years; and in the HGIEN group, there were 23 males, with an average age of $(61.6 \pm 9.2)$ years, and 26 females, with an average age of $(57.6 \pm 9.6)$ years. This study was approved by the ethics committee of Yancheng first people's Hospital (project number:: 2021-13364PR)

\subsection{Immunohistochemistry and evaluation}

Issues were fixed in formalin, paraffin-embedded, and cut into 4- $\mu \mathrm{m}$ sections. After dewaxing with xylene, dehydration with gradient ethanol, and washing with phosphate-buffered saline (PBS) 3 times, citrate-mediated high-pressure antigen retrieval was performed. Endogenous peroxide was inactivated with 3\% hydrogen peroxide solution, and 5\% bovine serum albumin (BSA) was used to block nonspecific staining sites. Primary antibody, anti-CKS2 (diluted at 1:200; Rabbit polyclonal 
antibody; Santa Cruz Biotechnology, Dallas, TX, USA) or anti-CD47 (diluted at 1:600; Rabbit polyclonal antibody; Santa Cruz Biotechnology, Dallas, TX, USA), were added, and the tissue sections were incubated at $4^{\circ} \mathrm{C}$ overnight. Secondary antibody was added, and the tissue sections were incubated at $37^{\circ} \mathrm{C}$ for $1 \mathrm{~h}$. The tissue sections were mounted and observed under an optical microscope. The results were evaluated by two pathologists without knowing any clinicopathological data. The evaluation criteria were as follows: the appearance of brownish yellow particles in the cell membrane or cytoplasm was regarded as positive staining. The staining results were scored based on the percentage of positive cells and staining intensity. The staining intensity score ranged from 0 to 3 , and the staining degree score ranged from 0 to $100 \%$. The final quantification of each stain was obtained by multiplying the two scores. If the evaluation results obtained by the two experts were consistent, the result was recorded as a final result; if the results were inconsistent, a third chief physician was invited to review the results, and the majority opinion was used as the final result.

\subsection{Follow-up}

The patients were followed up through outpatient visits and telephone interviews. Patients were followed up starting from the day of surgery and ending on the day of death or the last follow-up. Overall survival(OS) was defined as the time from the day of surgery to the day of death due to any reason. The follow-up ended on June 30, 2020, and the median follow-up time for the 126 patients was 46.5 months. Patients were followed up once every 1-3 months in the first 3 years, once every 6 months in the following 2 years, and once per year for the rest follow-up time. For the follow-up, tumor markers, i.e., carcinoembryonic antigen (CEA), alfa-fetoprotein (AFP), carbohydrate antigen (CA) 199, CA125, and CA724, were assessed; additionally, B-scan ultrasonography was performed every 3 months after gastrectomy and computed tomography (CT) or magnetic resonance imaging (MRI) was performed every 6 months after gastrectomy. There was no loss to follow-up in this study.

\subsection{Statistical analysis}

All statistical analyses were carried out using SPSS software. The chi-square test was used to analyze the correlation of CKS2 and CD47 expression with clinical data. The Student's t-test was 
used for comparisons. Correlation of CKS2 with CD47 staining scores was calculated by Pearson $\chi 2$ test. Survival curves were generated using the Kaplan-Meier method, and differences between curves were estimated by the log-rank test. The Cox multivariate proportional hazards regression model was used to determine the independent factors that influence prognosis based on the investigated variables. All reported $\mathrm{P}$ values were two-sided and $\mathrm{P}<0.05$ was considered statistically significant.

\section{Results}

2.1 Expression of CKS2 and CD47 in different gastric tissues

The positivity rates for CKS2 in benign gastric tumor tissues, LGIEN tissues, HGIEN tissues, and GC tissues gradually increased, i.e., $6.3 \%$ (2/32), 30.9\% (13/42), 38.8\% (19/49), and 60.3\% (76/126), respectively, as did the positivity rates for CD47, i.e., $18.8 \%$ (6/32), 38.1\% (16/42), $46.9 \%$ (23/49), and 65.9\% (83/126), respectively. Our results suggested that CKS2 and CD47 were high expression in gastric cancer tissues. The differences were statistically significant $\left(\chi^{2}=27.588\right.$, $\mathrm{P}=0.000 ; \chi^{2}=27.588, \mathrm{P}=0.000$ ), suggesting that $\mathrm{CKS} 2$ and CD47 may be associated with the development of GC (Fig. 1).
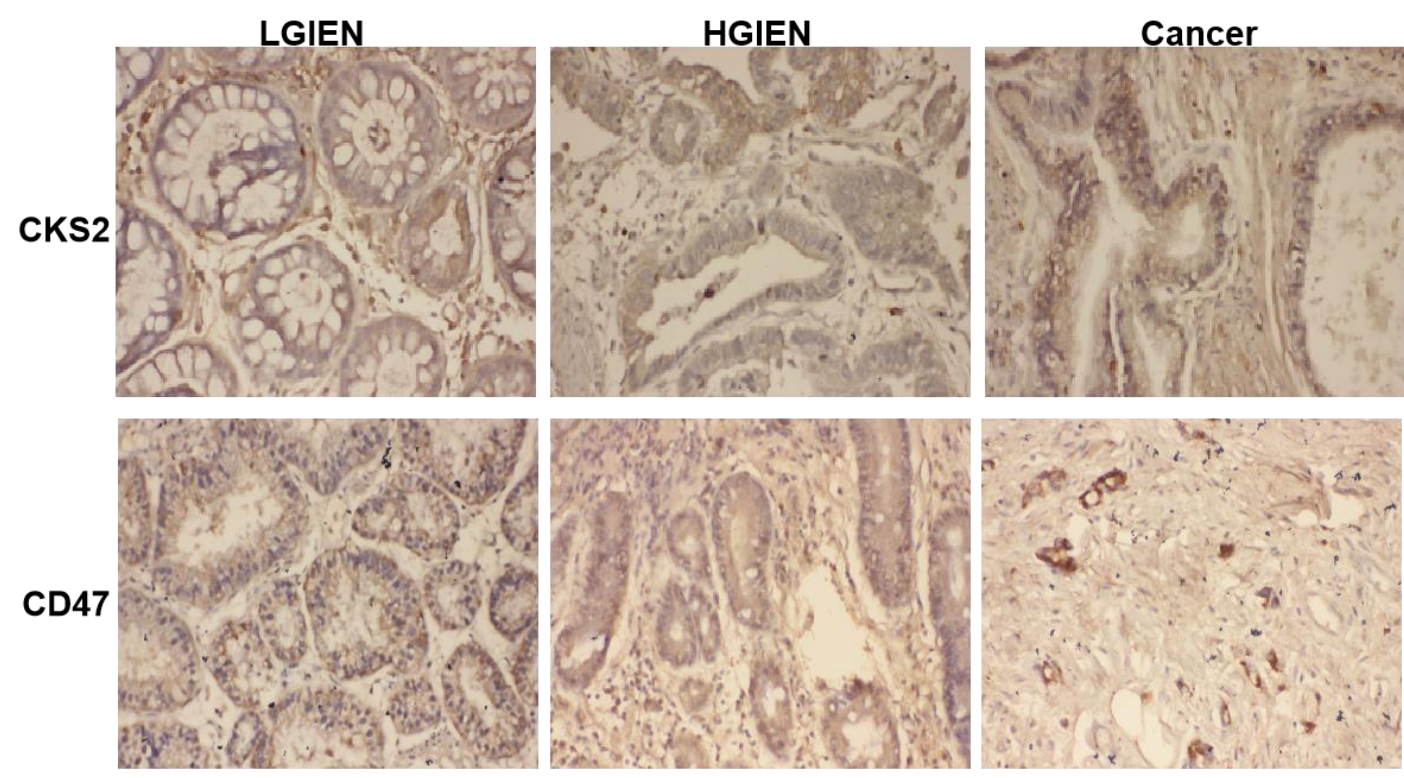

Fig .1 Expression of CKS2 and CD47 protein in different gastric tissues $(\times 200)$

2.2 Correlation of CKS2 and CD47 expression with clinicopathological features

To verify the functions of CKS2 and CD47 in GC, we correlated their expression with other 
widely recognized clinicopathologic features. Univariate analysis showed that high expression of CKS2 and CD47 were associated with tumor diameter, Lauren classification, number of lymph node metastases, and TNM stage of GC patients but were unrelated to patient gender, age, family history of GC, and histological type (Table 1).

Table 1 Correlation of CKS2 and CD47 expression with clinicopathological features

\begin{tabular}{|c|c|c|c|c|c|c|c|c|c|}
\hline \multirow[b]{2}{*}{ Index } & \multirow[b]{2}{*}{$\mathrm{n}$} & \multicolumn{2}{|c|}{ CKS2 express } & \multirow[b]{2}{*}{$\chi^{2}$} & \multirow[b]{2}{*}{$\mathrm{P}$} & \multicolumn{2}{|c|}{ CD47 express } & \multirow[b]{2}{*}{$\chi^{2}$} & \multirow[b]{2}{*}{$\mathrm{P}$} \\
\hline & & $\begin{array}{l}\text { negative } \\
(\mathrm{n}=50)\end{array}$ & $\begin{array}{l}\text { Positive } \\
(\mathrm{n}=76)\end{array}$ & & & $\begin{array}{c}\text { negative } \\
(\mathrm{n}=43)\end{array}$ & $\begin{array}{c}\text { Positive } \\
(\mathrm{n}=83)\end{array}$ & & \\
\hline Gender & & & & 0.590 & 0.443 & & & 1.711 & 0.191 \\
\hline Male & 78 & 33 & 45 & & & 30 & 48 & & \\
\hline Female & 48 & 17 & 31 & & & 13 & 35 & & \\
\hline Age & & & & 0.341 & 0.559 & & & 0.658 & 0.417 \\
\hline$<60$ & 62 & 23 & 39 & & & 19 & 43 & & \\
\hline$\geq 60$ & 64 & 27 & 37 & & & 24 & 40 & & \\
\hline Family history of GC & & & & 0.045 & 0.831 & & & 0.160 & 0.689 \\
\hline Yes & 29 & 12 & 17 & & & 9 & 20 & & \\
\hline No & 97 & 38 & 59 & & & 34 & 63 & & \\
\hline Tumor diameter(cm) & & & & 4.950 & 0.026 & & & 24.812 & 0.000 \\
\hline$<5$ & 73 & 35 & 38 & & & 38 & 35 & & \\
\hline$\geq 5$ & 53 & 15 & 38 & & & 5 & 48 & & \\
\hline Histological types & & & & 0.091 & 0.993 & & & 5.435 & 0.143 \\
\hline Tubular adenocarcinoma & 64 & 25 & 39 & & & 21 & 43 & & \\
\hline Papillary adenocarcinoma & 29 & 12 & 17 & & & 14 & 15 & & \\
\hline low adhesion adenocarcinoma & 26 & 10 & 16 & & & 5 & 21 & & \\
\hline Mucinous adenocarcinoma & 7 & 3 & 4 & & & 3 & 4 & & \\
\hline Lauren type & & & & 9.432 & 0.009 & & & 14.567 & 0.001 \\
\hline Intestinal type & 36 & 9 & 27 & & & 6 & 30 & & \\
\hline Diffuse type & 67 & 35 & 32 & & & 33 & 34 & & \\
\hline mixed type & 23 & 6 & 17 & & & 4 & 19 & & \\
\hline Number of lymph node metastases & & & & 7.770 & 0.005 & & & 10.181 & 0.000 \\
\hline$\leq 5$ & 69 & 35 & 34 & & & 32 & 37 & & \\
\hline$>5$ & 57 & 15 & 42 & & & 11 & 46 & & \\
\hline TNM stage & & & & 8.910 & 0.031 & & & 12.056 & 0.002 \\
\hline I & 37 & 20 & 17 & & & 19 & 18 & & \\
\hline II & 48 & 21 & 27 & & & 18 & 30 & & \\
\hline III & 41 & 9 & 32 & & & 6 & 35 & & \\
\hline
\end{tabular}


Spearman rank correlation analysis indicated that there was a moderate positive correlation between the expression levels of CKS2 and CD47 $(r=0.633, p=0.019)$. The correlation analysis of the immunohistochemical score also showed that there was a significant positive correlation between the immunohistochemical scores for CKS2 and CD47 ( $\mathrm{r}=0.625, \mathrm{P}=0.000)($ Table 2,Fig. $1)$.

Table 2 Correlation Analysis of CKS2、CD47 Expression Intensity

\begin{tabular}{ccccc}
\hline \multirow{2}{*}{ CKS2 express } & \multicolumn{2}{c}{ CD47 express } & & P \\
\cline { 2 - 3 } & negative & Positive & & 0.019 \\
\hline negative & 34 & 15 & 0.633 & 0.0 \\
Positive & 13 & 64 & & \\
\hline
\end{tabular}

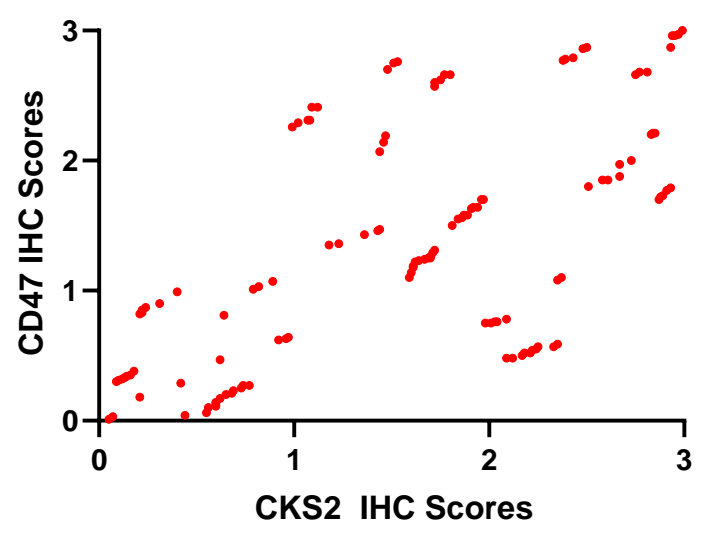

Fig .2 Correlation analysis of CKS2 and CD47 immunohistochemical scores

\subsection{High expression of CKS2 and CD47 predict poor prognosis}

The median follow-up time for the 126 patients was 46.5 months, and the overall survival rate was $40.5 \%$ (51/126). The overall survival rates for the patients in the CKS2(+) and CKS2(-) groups were $25.0 \%$ (19/76) and $64.0 \%$ (32/50), respectively, and the overall survival rate for the patients in the CKS2 $(+)$ group was significantly poor $(\chi 2=15.67, \mathrm{P}=0.000)$. The overall survival rates for the patients in CD47 (+) and CD47 (-) groups were $30.1 \%$ (25/83) and 60.5\% (26/43), respectively, and the overall survival rate for the patients in the CD47(-) group was significantly poor $(\chi 2=14.14, \mathrm{P}=$ 0.000). Based on CKS2 and CD47 expression, the patients were divided into 4 groups: CKS2(+)CD47(+) group(n=65), CKS2(+)CD47(-) group(n=12), CKS2(-)CD47(+) group(n = 14), and CKS2(-)CD47(-) group $(\mathrm{n}=35)$; the overall survival rates were $20.0 \%(13 / 65), 58.3 \%(7 / 12)$, $57.1 \%(8 / 14), 65.7 \%(23 / 35)$, respectively, and the differences were statistically significant $\left(\chi^{2}=\right.$ 
$18.14, \mathrm{P}=0.000)$. The prognosis of patients in $\mathrm{CKS} 2(+) \mathrm{CD} 47(+)$ group was significantly poor (Fig.

$3)$.
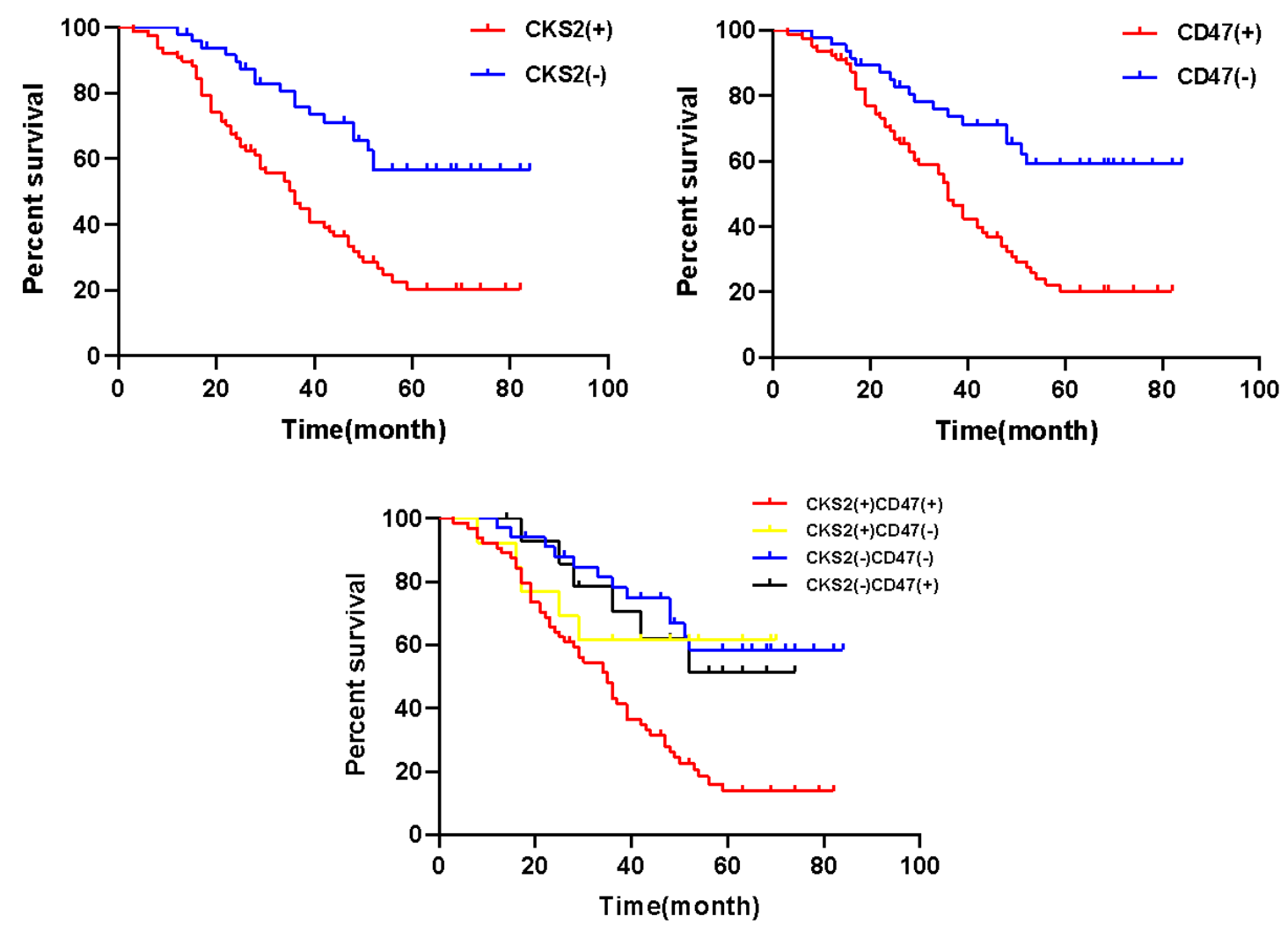

Fig.3 Effect of CKS2 and CD47 expression on overall survival rate of GC patients

2.5 Cox multivariate prognosis analysis of GC patients

Cox multivariate survival analysis showed that TNM stage, lymph node metastasis, CKS2 expression, and CD47 expression were independent risk factors affecting the OS of patients $(\mathrm{P}<$ 0.05). Table 3.

Table 3 Cox Multivariate survival analysis

\begin{tabular}{ccccccc}
\hline Index & $\beta$ & SE & Wald & P & OR & 95\%CI \\
\hline Gender & 0.044 & 0.017 & 0.549 & 0.440 & 0.568 & $0.719 \sim 0.936$ \\
Age & 0.031 & 0.010 & 0.338 & 0.213 & 0.984 & $0.821 \sim 1.187$ \\
Tumor site & -0.039 & 0.041 & 0.238 & 0.114 & 1.052 & $0.269 \sim 1.485$ \\
Tumor diameter & -0.044 & 0.085 & 1.152 & 0.229 & 0.931 & $0.422 \sim 1.968$ \\
Lauren type & 0.038 & 0.048 & 0.207 & 0.536 & 1.007 & $0.963 \sim 1.841$ \\
TNM stage & 0.631 & 0.249 & 6.487 & 0.011 & 2.865 & $1.158 \sim 3.096$ \\
lymphatic metastasis & 0.839 & 0.228 & 13.698 & 0.013 & 2.396 & $1.496 \sim 3.584$ \\
CKS2 express & 0.487 & 0.058 & 5.138 & 0.003 & 2.130 & $1.074 \sim 3.639$ \\
CD47 express & 0.465 & 0.017 & 6.015 & 0.015 & 2.654 & $1.152 \sim 5.874$ \\
\hline
\end{tabular}




\section{Discussion}

In mammals, CKS1 and CKS2, are composed of 79 amino acids, and the homology between their protein sequences is $81 \%$. Richardson et a ${ }^{[9]}$ first discovered the genes in 1990 and established that human CKS1 is located on chromosome 8q21 and CKS2 is located on chromosome 9q22. Studies have shown that both CKS1 and CKS2 are involved in the regulation of cell proliferation. As a required component of SCFSkp2-mediated ubiquitination degradation of p27, CKS1 is mainly involved in the regulation of cell cycle G1/S conversion, while CKS2 is crucial for cell cycle G2/M conversion $^{[10]}$. CKS family members are closely related to cancer development. The protein expression levels of CKS1 and CKS2 in various cancer tissues, including breast cancer ${ }^{[5]}$, cervical cancer $^{[11]}$, ovarian cancer ${ }^{[12]}$, prostate cancer, and hepatocellular carcinoma ${ }^{[13]}$, are increased and closely related to the poor prognosis of patients. To confirm the correlation between CKS2 expression and the occurrence of GC, this study examined CKS2 protein expression in GC tissues. The results indicated that CKS2 expression in cancer tissues was significantly higher than that in noncancerous tissues, a result that was consistent with those reported in the literatures. CD47 is a widely expressed antigen and is usually highly expressed in tumor cells. Studies have shown that CD47 is highly expressed on the surface of tumor cells of various cancers, including ovarian cancer $^{[14]}$, breast cancer ${ }^{[15]}$, osteosarcoma ${ }^{[16]}$, small cell lung cancer ${ }^{[17]}$ and liver cancer ${ }^{[18]}$. Yoshida et $\mathrm{al}^{[19]}$. found that among $115 \mathrm{GC}$ patients, the positive expression rate of $\mathrm{CD} 47$ was $49.5 \%$ (57/115). Gan et al. showed that the positive expression rate of CD47 in $80 \mathrm{GC}$ tissues was 51.85\%, while that in normal paracancerous tissues was $36.84 \%$. In this study, the positivity rate for CD47 in GC tissues was 65.9\% (83/126), a result that was consistent with the reported rate for CD47.

The high CD47 expression in tumor cells can protect tumor cells from being phagocytosed by macrophages, thus contributing to the further development of tumors. Therefore, CD47 expression is closely related to the clinicopathological factors of cancer patients, but the correlations are different. Liu et al ${ }^{[20]}$. showed that in ovarian cancer, CD47 expression was closely related to the degree of ovarian tissue differentiation and the pathological stage: the higher the CD47 expression, the worse the differentiation degree of ovarian cancer tissues, and the more advanced the pathological stage. In addition, a report on the correlation between CD47 and bladder cancer stage showed that high CD47 expression was closely related to the T stage of bladder cancer: the higher 
CD47 expression, the higher the T stage of bladder cancer. However, Sudo et al ${ }^{[21]}$. found that CD47 was highly expressed in highly differentiated gastric adenocarcinoma, slightly less expressed in moderately differentiated gastric adenocarcinoma compared to highly differentiated gastric adenocarcinoma, and almost not expressed in poorly differentiated gastric adenocarcinoma. This observation is inconsistent with the conclusion of this study. This study found that CD47 protein expression had significant adverse effects on tumor diameter, number of lymph node metastases, and TNM stage.

It has been reported that $\mathrm{CKS} 2$ expression is related to the clinicopathologic features of tumors and can be used as a prognostic factor to determine the survival time of patients. CKS2 expression in colorectal cancer has a significantly negative correlation with the degree of tumor differentiation, and CKS2 is also highly expressed in GC and correlated with the degree of tumor differentiation. Liu et al. showed that CKS2 gene expression in GC tissues $(0.97 \pm 0.16)$ was higher than that in paracancerous tissues $(0.38 \pm 0.11)$ and that the expression of CKS2 protein in GC tissues was closely related to Lauren classification, depth of tumor invasion, lymph node metastasis, and TNM stage. Bioinformatics analysis results further confirmed that the high expression of CKS2 protein in GC tissues was closely related to the occurrence and development of GC and can be used as an independent factor to evaluate the prognosis of patients with GC. This study found that CKS2 protein expression had significantly adverse effects on tumor diameter, number of lymph node metastases, and TNM stage, a result that is consistent with those reported in the literature.

Approximately $80 \%$ of tumor cell recurrence and metastasis occur within three years after surgery, and another $10 \%$ occur within 5 years after radical surgery. Therefore, it is generally accepted in the clinic that if a tumor does not relapse within 5 years after surgery, the probability of recurrence is very low and negligible. Shi et al ${ }^{[22]}$. showed that high CD47 expression was positively correlated with TNM stage, the incidence of distant metastasis, and the mortality rate of melanoma patients and that high CD47 expression was an independent prognostic indicator of overall survival and progression-free survival in GC patients. However, the relationship between CD47 expression and the prognosis of patients with GC is under debate. Yoshida et al ${ }^{[19]}$. showed that the overall survival rate for CD47(+) patients was significantly lower than that for CD47(-) patients and that CD47 expression was considered a risk factor for a poor prognosis of patients with GC. However, Sudo et al ${ }^{[21]}$. showed that CD47 expression in primary tumors was unrelated to any 
clinicopathological factor or prognosis; these results indicated that there might be posttranscriptional differences, such as protein degradation or transport damage, that lead to high CD47 protein expression. Yu et al ${ }^{[23]}$. showed that CKS2 expression levels in colorectal cancer tissues were significantly higher than those in paracancerous tissues and that CKS2 protein was highly expressed in colorectal cancer and correlated with the clinicopathological characteristics of tumors; they concluded that CKS2 expression can be utilized as a new molecular marker and therapeutic target for colorectal cancer. Our study showed that the 5-year survival rate of patients in the CD47(-)CKS2(-) group was significantly better than that of patients in the CD47(+)CKS2(+) group; these results indicated that the higher the expression levels of CD47 and CKS2, the worse the prognosis of patients with GC. Further multivariate analysis using a Cox proportional hazard model showed that CD47 and CCK2 expression levels were independent risk factors affecting the prognosis of patients with GC. Pathological tumor-node-metastasis (pTNM) staging is critical for determining the prognosis of GC and selecting clinical treatments.

In summary, this study found that CKS2 and CD47 expression levels were related to the clinicopathological features of GC and may play an important role in the occurrence and development of GC through a synergistic effect. These results indicated that the CKS2 and CD47 could play important regulatory roles in the biological behavior of GC. This study provides a theoretical basis for the use of CKS2 and CD47 protein as novel molecular markers and therapeutic targets for GC.

\section{Reference}

[1] Torre LA, Bray F, Siegel RL, et al. Global cancer statistics, 2012[J]. CA: a cancer journal for clinicians, 2015, 65(2): 87-108.

[2] Du Y, Zhu H, Liu J, et al. Consensus on eradication of Helicobacter pylori and prevention and control of gastric cancer in China (2019, Shanghai)[J]. Journal of gastroenterology and hepatology, 2020, 35(4): 624-629.

[3] Siegel R, Ma J, Zou Z, et al. Cancer statistics, 2014[J]. CA: a cancer journal for clinicians, 2014, 64(1): 9-29. 
[4] Seo S, Ryu M-H, Park YS, et al. Loss of HER2 positivity after anti-HER2 chemotherapy in HER2-positive gastric cancer patients: results of the GASTric cancer HER2 reassessment study 3 (GASTHER3)[J]. Gastric Cancer, 2019, 22(3): 527-535.

[5] Huang $\mathrm{N}, \mathrm{Wu} \mathrm{Z}$, Hong $\mathrm{H}$, et al. Overexpression of CKS2 is associated with a poor prognosis and promotes cell proliferation and invasion in breast cancer[J]. Molecular medicine reports, 2019, 19(6): 4761-4769.

[6] Martinsson-Ahlzén H-S, Liberal V, Grünenfelder B, et al. Cyclin-dependent kinase-associated proteins $\mathrm{Cks} 1$ and $\mathrm{Cks} 2$ are essential during early embryogenesis and for cell cycle progression in somatic cells[J]. Molecular and cellular biology, 2008, 28(18): 5698-5709.

[7] Candas-Green D, Xie B, Huang J, et al. Dual blockade of CD47 and HER2 eliminates radioresistant breast cancer cells[J]. Nature communications, 2020, 11(1): 1-15.

[8] Yang K, Xu J, Liu Q, et al. Expression and significance of CD47, PD1 and PDL1 in T-cell acute lymphoblastic lymphoma/leukemia[J]. Pathology-Research and Practice, 2019, 215(2): 265-271.

[9] Richardson HE, Stueland CS, Thomas J, et al. Human cDNAs encoding homologs of the small p34Cdc28/Cdc2-associated protein of Saccharomyces cerevisiae and Schizosaccharomyces pombe[J]. Genes \& Development, 1990, 4(8): 1332-1344.

[10] Zhao H, Iqbal NJ, Sukrithan V, et al. Targeted inhibition of the E3 ligase SCFSkp2/Cks1 has antitumor activity in RB1-deficient human and mouse small-cell lung cancer[J]. Cancer research, 2020, 80(11): 2355-2367.

[11] Jonsson M, Fjeldbo CS, Holm R, et al. Mitochondrial function of CKS2 oncoprotein links oxidative phosphorylation with cell division in chemoradioresistant cervical cancer[J]. Neoplasia, 2019, 21(4): 353-362.

[12] $\mathrm{Xu} \mathrm{JH}$, Wang Y, Xu D. CKS2 promotes tumor progression and metastasis and is an independent predictor of poor prognosis in epithelial ovarian cancer[J]. European review for medical and pharmacological sciences, 2019, 23(8): 3225-3234.

[13] Li Z, Xue TQ, Yang C, et al. EGFL7 promotes hepatocellular carcinoma cell proliferation and inhibits cell apoptosis through increasing CKS2 expression by activating Wnt/ $\beta$-catenin signaling[J]. Journal of cellular biochemistry, 2018, 119(12): 10327-10337.

[14] Wang H, Tan M, Zhang S, et al. Expression and significance of CD44, CD47 and c-met in ovarian clear cell carcinoma[J]. International journal of molecular sciences, 2015, 16(2): 3391-3404.

[15] Zhang H, Lu H, Xiang L, et al. HIF-1 regulates CD47 expression in breast cancer cells to promote evasion of phagocytosis and maintenance of cancer stem cells[J]. Proceedings of the National Academy of Sciences, 2015, 112(45): E6215-E6223.

[16] Mohanty S, Yerneni K, Theruvath JL, et al. Nanoparticle enhanced MRI can monitor macrophage response to CD47 mAb immunotherapy in osteosarcoma[J]. Cell death \& disease, 2019, 10(2): 1-14.

[17] Zhang X, Wang Y, Fan J, et al. Blocking CD47 efficiently potentiated therapeutic effects of anti-angiogenic therapy in non-small cell lung cancer[J]. Journal for immunotherapy of cancer, 2019, 7(1): 1-11. 
[18] Rodríguez MM, Fiore E, Bayo J, et al. 4Mu decreases CD47 expression on hepatic cancer stem cells and primes a potent antitumor $\mathrm{T}$ cell response induced by interleukin-12[J]. Molecular Therapy, 2018, 26(12): 2738-2750.

[19] Yoshida K, Tsujimoto H, Matsumura K, et al. CD 47 is an adverse prognostic factor and a therapeutic target in gastric cancer[J]. Cancer medicine, 2015, 4(9): 1322-1333.

[20] Liu R, Wei H, Gao P, et al. CD47 promotes ovarian cancer progression by inhibiting macrophage phagocytosis[J]. Oncotarget, 2017, 8(24): 39021.

[21] Sudo T, Takahashi Y, Sawada G, et al. Significance of CD47 expression in gastric cancer[J]. Oncology letters, 2017, 14(1): 801-809.

[22] Shi M, Gu Y, Jin K, et al. CD47 expression in gastric cancer clinical correlates and association with macrophage infiltration[J]. Cancer Immunology and Immunotherapy, 2021, (10060): 1-10.

[23] Yu MH, Luo Y, Qin SL, et al. Up-regulated CKS2 promotes tumor progression and predicts a poor prognosis in human colorectal cancer[J]. Am J Cancer Res, 2015, 5(9): 2708-2718. 